\title{
Acknowledgement to Reviewers of Minerals in 2015
}

\author{
Minerals Editorial Office \\ Published: 21 January 2016 \\ MDPI AG, Klybeckstrasse 64, CH-4057 Basel, Switzerland; minerals@mdpi.com
}

The editors of Minerals would like to express their sincere gratitude to the following reviewers for assessing manuscripts in 2015.

We greatly appreciate the contribution of expert reviewers, which is crucial to the journal's editorial decision-making process. Several steps have been taken in 2015 to thank and acknowledge reviewers. Good, timely reviews are rewarded with a discount off their next MDPI publication. By creating an account on the submission system, reviewers can access details of their past reviews, see the comments of other reviewers, and download a letter of acknowledgement for their records. In addition, MDPI has launched a collaboration with Publons, a website that seeks to publicly acknowledge reviewers on a per journal basis. This is all done, of course, within the constraints of reviewer confidentiality. Feedback from reviewers shows that most see their task as a voluntary and mostly unseen work in service to the scientific community. We are grateful to our reviewers for the contribution they make.

Abou-Taouk, Abdallah
Abreu, Neyda
Alexandre, Paul
Alves, Carlos
Ametov, Igor
Atlas, Zachary
Atwood, David A.
Ávila, Paula F.
Aydin, Isil
Bahri, Parisa Arabzadeh
Baldwin, Susan A.
Bayramgil, Nursel Pekel
Benardos, Andreas G.
Bhattacharyya, Amrita
Binnemans, Koen
Boni, Maria
Botas, Cristina
Bradshaw, Alex M.
Bräuer, Suzanna
Brierley, Corale
Briggs, Derek
Buckley, Alan
Budzyn, Bartosz
Buhl, Elmar
Busacca, Concetta
Cai, Jun

Cai, Yaoping
Canbulat, Ismet
Chaffee, Alan
Chagnes, Alexandre
Chapman, Robert
Chapon, Virginie
Charro, Elena
Chen, Rui
Chen, Zhanming
Chen, Liangbiao
Chiang, Pen-chi
Chiang, Yi Wai
Choi, Man Sik
Christanis, Kimon
Christmann, Patrice
Cisternas, Luis A.
Conko, Kathryn
Cowan, Ellen A.
Dai, Shifeng
Dawson, Grant
De la Piscina, Pilar Ramirez
De La Rosa, Jesús
Deglon, David
Di Benedetto, Francesco
Dickinson, Jamie
Dittrich, Maria

\author{
Dobrica, Elena \\ DoradoCastaño, Antonio David \\ Drost, Kerstin \\ Dzade, Nelson \\ Đurasević, Mirjana \\ Eble, Cortland F. \\ Edraki, Mansour \\ Ehrlich, Hermann \\ Eisele, Timothy C. \\ Eliasson, Jens \\ Errandonea, Daniel \\ Esquivias, Luis \\ Ettler, Vojtech \\ Farrauto, Robert J. \\ Farrokhpay, Saeed \\ Finkelman, Robert B. \\ Fitzpatrick, Robert \\ Fonkwe, Merline \\ Francis, Arokiasami \\ Gadikota, Greeshma \\ Geboy, Nicholas J. \\ Ghosh, Tathagata \\ Giudici, Giovanni De \\ Golev, Artem \\ Graedel, Thomas \\ Grieco, Giovanni
}




Gutzmer, Jens
Hadler, Kathryn
Haeri Ardakani, Omid
Hellevang, Helge
Hofmann, Annette
Holuszko, Maria
Honaker, Rick
Hower, Jim
Huang, Chin-Pao
Jameson, Graeme
Jamieson, John
Janeczek, Janusz
Jang, Young-nam
Jilbert, Tom
Jones, Tristan
Julcour-Lebigue, Carine
Katsoyiannis, Ioannis A.
Keenan, Sarah W.
Kessler, Vadim
Khandelwal, Manoj
Kim, Eunhye
Klein, Bern
Kojovic, Toni
Kolker, Allan
Koshy, Pramod
Kosinski, Pawel
Koukouzas, Nikolaos
Kowalczuk, Przemyslaw B.
Kowalski, Nicole
Kremenovic, Aleksandar
Kuhar, Laura
Kutschke, Sabine
Lacinska, Alicja Magdalena
Lancaster, Penny
Large, David
Laskowski, Janusz
Laumonier, Mickaël
Le Guillou, Corentin
Lee, Sihyun
Lefticariu, Liliana
Lehmann, Bernd
Li, Hong-Yu
Li, Xueliang
Li, Songnian
Liu, Don
Liu, Wei Victor
Liu, Shi Qiang
Lu, Candy (Ying)
Luo, Yi

Ma, Tingguang

Marshall, Chris

Martins, José Inácio

Mayes, Richard

McDonald, Robbie

Mclemore, Virginia $\mathrm{T}$.

Meegoda, Jay N.

Mei, Yuan

Menéndez-Aguado, J.M.

Mercier, Patrick H.J.

Milke, Ralf

Mitra, Rudrajit

Mohanarangam, Krishna

Mojtabai, Navid

Mongelli, Giovanni

Moreno, Natalia

Murakami, Shinsuke

Murphy, Melissa J.

Nalla, Venkatram

Neupane, Ghanashyam

Noble, Aaron

Nugteren, Henk

Oger, Phil M.

Oosthuizen, Jacques

Opluštil, Stanislav

Pan, Wei-Ping

Parat, Fleurice

Pearce, Julie

Peng, Felicia F.

Phan, Chi

Pietrzak, Robert

Pirard, Cassian

Potra, Adriana

Power, Ian M.

Prasad, Vinay

Quirt, David

Ralston, Jonathon C.

Ram, Rahul

Rashidi Nejad, Farshad

Reinmöller, Markus

Reith, Frank

Ren, Deyi

Renforth, Phil

Retallack, Gregory J.

Reynier, Nicolas

Richard, Antonin

Rose, Andrew

Rose, Neil L

Ruhl, Laura
Saini-Eidukat, Bernhardt

Santos, Rafael M.

Sarli, Valeria Di

Sasaoka, Takashi

Sasmito, Agus Pulung

Schiffbauer, James

Schijf, Johan

Schmalenberger, Achim

Schobert, Harold H.

Senko, John M.

Shang, Zhi

Shang, Helen

Shaw, Richard

Singh, Dhananjay

Skinner, William

Spears, David Alan

Stace, Rod

Stamatakis, Michail

$\mathrm{Su}, \mathrm{Bin}$

Sun, Henghu

Suzuki, Shigeru

Taheri, Abbas

Tanaka, Akira

Tarshizi, Ebrahim Karimi

Tavares, Luís Marcelo

Thien, Bruno

Tomita, Koji

Toner, Brandy

Tropper, Peter

Ullah, Shatif

Vajda, Vivi

Valentim, Bruno

Vallianatos, Filippos

Vaughan, James

Vink, Sue

Volzone, Cristina

Wang, Shanyong

Ward, Colin

Watling, Helen

Widiatmojo, Arif

Witt, Peter

$\mathrm{Xu}$, Guang

Yalcin, Turgut

Yalçın, Hüseyin

Yeh, Sonia

Zanin, Massimiliano

Zhang, Kuangyuan

Zheng, David

Zhou, Hui 